\title{
Privatisierung der Infrastruktur und Raumordnungspolitik
}

\section{René L. Frey, Basel}

Wichtige Bereiche der Infrastruktur sind in den letzten Jahren liberalisiert, dereguliert oder privatisiert worden. Welche Konsequenzen hat dies für die Raumordnungspolitik, die sich in der Vergangenheit stark der Infrastruktur als Instrument zur Beeinflussung der räumlichen Struktur (Raumplanung) und Förderung von Berg- und wirtschaftlichen Problemgebieten (Regionalpolitik) bedient hat? Verliert die Raumordnungspolitik durch diese neue Entwicklung an Wirksamkeit? Wie könnte sie sich gegebenenfalls neu ausrichten?

Im Folgenden wird - vor allem gestützt auf zwei kürzlich erschienene Arbeiten (INFras 1998, Frey 1999) gezeigt,

- warum es gerade in den neunziger Jahren zu einer Liberalisierungswelle gekommen ist.

- welche Infrastrukturbereiche von der Liberalisierung betroffen sind.

- worin sich die Liberalisierung äussert.

- wie sich die Liberalisierung spezifisch in den Bereichen Schienenverkehr, Post, Telekommunikation und Elektrizität präsentiert, welche Schritte bereits getan worden sind und welche noch bevorstehen.

- welche Konsequenzen sich daraus für die Raumordnungspolitik ergeben.

\section{Infrastruktur als Instrument der Raumordnungs- politik}

Die vier Bereiche Verkehr, Energie, Telekommunikation und, etwas weniger eindeutig, Post lassen sich unter der Bezeichnung Netzinfrastruktur zusammenfassen. Davon zu unterscheiden ist die Punktinfrastruktur, wie sie zum Beispiel für Schulen, Spitäler, Theater oder Museen, aber auch für Verwaltungseinrichtungen und die Feuerwehr charakteristisch ist. Im Gegensatz zur Netzinfrastruktur, bei der am Ort der Nachfrager oder in deren Nähe Infrastrukturleistungen zur Verfügung gestellt werden (ÖV-Haltestelle, Elektrizität, Telefonanschluss, Postzustellung usw.), begeben sich bei der Punktinfrastruktur die Nachfrager zum Ort des Angebots der Infrastrukturleistungen.
In der bisherigen Raumordnungspolitik wurden beide Arten von Infrastrukturen eingesetzt:

- Beim Bundesgesetz über Investitionshilfe für Berggebiete (Investitionshilfegesetz oder kurz IHG) aus dem Jahre 1974 liegt das Schwergewicht vor allem auf der kleinräumigen Punktinfrastruktur. Durch den Bund können Projekte der Bereiche regionaler und lokaler Verkehr, Versorgung und Entsorgung, Schutz vor Elementarschäden, Bildung, Gesundheit, Erholung/Sport/Freizeit, Kultur, öffentliche Verwaltung und Konsummöglichkeiten gefördert werden (vgl. Frey 1979). Bis Ende 1999 wurden im Rahmen des IHG 7056 Projekte mit Gesamtkosten von 16,5 Milliarden Franken durch den Bund mit 1,7 Milliarden Franken Darlehen und 720 Millionen Franken Zinskostenbeiträgen unterstützt (www.seco.admin.ch/standort/).

- Bei denjenigen Infrastrukturbereichen, die gegenwärtig liberalisiert werden, handelt es sich um die grossräumige Netzinfrastruktur. Ihr Einsatz für Zwecke der Raumordnungspolitik ist nicht quantitativ zu erfassen; er nimmt unterschiedlichste Formen an. Das gemeinsame Merkmal bestand in der Vergangenheit in Auflagen des Staates an die Adresse der Infrastrukturbetriebe, gewisse Leistungen im öffentlichen Interesse (z.B. Umweltschutz, Landesverteidigung) oder zugunsten bestimmter, beispielsweise peripherer, Gebiete auch dann zu erbringen, wenn diese betriebswirtschaftlich nicht zu rechtfertigen sind. Die wohl besten Beispiele für derartige gemeinwirtschaftliche Leistungen sind im öffentlichen Verkehr die Fahrplanpflicht (z.B. Einstundentakt) und die Tarifpflicht (z.B. günstige Transportgebühren). Im Energiebereich wurde Regionalpolitik vor allem über die Wasserzinsen und die Gratisenergie betrieben.

\section{Paradigmawechsel im Infrastrukturbereich}

Mit der Privatisierung weiter Bereiche der Netzinfrastruktur ist diese Art der Raumordnungspolitik nunmehr in Frage gestellt. Unternehmungen, die sich im internationalen Wettbewerb behaupten müssen, kann nicht zugemutet werden, derartige gemeinwirtschaftliche Leistungen zu erbringen - ausser die dadurch entstehenden Zusatzkosten werden durch den Staat abgegolten.

Um die neue Stituation einschätzen zu können, muss untersucht werden, warum die Netzinfrastruktur rund 
100 Jahre lang in staatlicher Hand war - und folglich auch in den Dienst staats- und raumordnungspolitischer Ziele gestellt werden konnte - und warum dies heute nur noch bedingt, in Zukunft möglicherweise überhaupt nicht mehr der Fall ist.

\subsection{Regulierung und Verstaatlichung zur Verhinde- rung natürlicher Monopole}

Das wirtschaftliche Hauptmerkmal der Netzinfrastruktur sind ausgeprägt sinkende Durchschnittskosten und Grössenvorteile. Je mehr Einheiten einer Infrastrukturleistung produziert werden, desto kostengünstiger kann dies geschehen. Daraus entsteht ein Zwang zur Grösse. Wenn sich aber der grösste Produzent oder Anbieter durchsetzt und seine Konkurrenten vom Markt verdrängt, kommt der Wettbewerb zum Erliegen. Ökonomen sprechen von ruinöser Konkurrenz (vgl. Kaufer 1981). Um dieses Problem von sogenannten natürlichen Monopolen zu lösen, sind zwei Arten von Gegenmassnahmen ergriffen worden (vgl. VON WEIZSÄCKER 1982, SHLEIFER 1998):

- In den USA waren die Infrastrukturunternehmungen (public utilities) seit jeher privat. Sie wurden jedoch staatlich kontrolliert, und zwar durch

- wettbewerbspolitische Massnahmen (Verbot von Fusionen, Antitrustgesetzgebung).

- Regulierung privater Monopole (staatliche Investitions-, Angebots- und Preisvorschriften).

- In Europa - auch in der Schweiz - wurde ein anderer Weg gewählt:

- die Verstaatlichung (Überführung von Infrastrukturunternehmungen in öffentliches Eigentum bzw. Gründung als staatliche oder gemischtwirtschaftliche Unternehmungen).

- verbunden mit einer spezialgesetzlichen Normierung der staatlichen Monopole.

Bis vor wenigen Jahrzehnten war sowohl in der Wirtschaftswissenschaft als auch in der Praxis unbestritten, dass Regulierung oder Verstaatlichung im Falle von natürlichen Monopolen unerlässlich sind. Seit der Ära Carter (besonders aber unter Reagan und Thatcher) wird diese Sicht nun aber in Frage gestellt und sind Massnahmen zur Korrektur von Regulierung und Verstaatlichung eingeleitet worden ( $\mathrm{vgl}$. KAHN 1988). Mit einer zeitlichen Verzögerung ist auch Kontinentaleuropa von diesem Sog ergriffen worden. Während in den USA das gleiche Phänomen unter der Bezeichnung Deregulierung (deregulation) läuft, steht in Europa die Privatisierung im Vordergrund.

\subsection{Liberalisierung - Oberbegriff für Privatisierung und Deregulierung}

Als Oberbegriff für «Deregulierung» (in den USA) und «Privatisierung» (in Europa) wird im Folgenden «Liberalisierung» verwendet. In allen Fällen geht es darum, in öffentlichen oder öffentlich kontrollierten Wirtschaftsbereichen zur Effizienzsteigerung und Innovationsförderung mehr privatwirtschaftliche Eigentumselemente und/oder mehr marktwirtschaftliche Steuerungselemente einzuführen.

Warum werden heute Regulierung und Verstaatlichung rückgängig gemacht? Bei der Beantwortung dieser Frage wird im Folgenden vor allem auf Europa und die Schweiz - und damit auf den Fall der Privatisierung - eingegangen.

\subsubsection{Effizienzprobleme der bisherigen politisch-administrativen Steuerung}

Unter den alten Bedingungen wurden in Europa die Verkehrs-, Post-, Telekommunikations- und EnergieVersorgungsbetriebe weitgehend politisch-administrativ geführt. Dadurch fehlten der Spielraum und die Anreize zu unternehmerischem Handeln. Leicht überzeichnet lässt sich dieses alte System wie folgt umschreiben:

- Das Parlament legt in Gesetzen - explizit oder implizit sanktioniert durch die Stimmbürger - fest, welche Leistungen die öffentlichen Infrastrukturbetriebe zu erbringen haben.

- Das Parlament bestimmt im Rahmen der Budgetierung, welche Mittel jeweils für ein Kalenderjahr zur Verfügung stehen und wofür diese zu verwenden sind (sog. Inputsteuerung).

- Das Bestreben der «Infrastrukturbürokratie» ist darauf ausgerichtet,

- alle bewilligten Mittel vollständig auszugeben (sog. «Dezemberfieber»).

- möglichst hohe Budgetanträge einzureichen, weil das Prestige einer Abteilung und ihres Chefs (und dessen Einkommen) massgeblich von der Höhe des Budgets abhängt.

- Die einzelnen Beamten tun gut daran, die rechtlichen Vorschriften buchstabengetreu zu befolgen. Dann kann ihnen nichts passieren. Wirtschaftliche Aufgabenerfüllung wird nicht honoriert.

- Die Bedürfnisse der Kunden sind sekundär. Es ist nicht Sache der Beamten, sie zu berücksichtigen. Man geht vielmehr davon aus, dass dies Aufgabe der politischen Instanzen ist. Marketing ist unter den alten Bedingungen ein Fremdwort. 
Die politisch-administrative Steuerung der Infrastrukturbereiche hatte vier unerwünschte Konsequenzen:

- Die öffentliche Verwaltung beziehungsweise die öffentlichen Unternehmungen stellten nicht diejenigen Waren und Dienstleistungen her, welche die Bevölkerung und die Unternehmungen wollten (gesamtwirtschaftliche Ineffizienz).

- Das Angebot entsprach in qualitativer Hinsicht nicht den Bedürfnissen von Bevölkerung und Wirtschaft (qualitative Inefffizienz).

- Die öffentlichen Leistungen wurden nicht zu den tiefstmöglichen Kosten produziert (betriebliche Ineffizienz).

- Weil der Konkurrenzdruck fehlte, waren die Anreize für Produkt- und Prozessinnovationen äusserst schwach (dynamische Ineffizienz).

\subsubsection{Veränderte Rahmenbedingungen: Globalisie- rung und Standortwettbewerb}

Diese Ineffizienzen wurden akzeptiert, solange die Volkswirtschaften weitgehend nationale Volkswirtschaften waren. Mit den verschiedenen GATT-Runden, der Gründung der WTO sowie der Schaffung des einheitlichen europäischen Binnenmarktes durch die EU sind heute die nationalen Grenzen für Waren, Dienstleistungen, Personen und Kapital durchlässiger geworden. Hinzu kommen stark sinkende Transportkosten und technologische Neuerungen vor allem in Informatik und Telekommunikation, die ihrerseits die Welt kleiner und die einzelnen Märkte grösser machen.

Das neue wirtschaftspolitische Paradigma lässt sich umschreiben mit: Harmonisierung und Öffnung der Märkte. Diese als Globalisierung bezeichnete Entwicklung hat den Wettbewerb zwischen Unternehmungen gewaltig verschärft. Diese mussten sich der neuen Situation durch Rationalisierung und Innovation, aber auch durch Standortverlagerung anpassen. Es gelang ihnen, glaubhaft nachzuweisen, dass Abwanderung von Firmen, Verlagerung von Betriebsstätten und Verlust an Arbeitsplätzen in den alten Industrieländern nur verhindert werden können, wenn die lokale Wirtschaft, das Gewerbe und der Staat die Anpassungslasten mittragen. Das heisst: Rahmenbedingungen verbessern, billiger produzieren sowie Kosten, Preise und Steuern senken. In zunehmendem Ausmass ist auf diese Weise aus dem Wettbewerb zwischen privaten Unternehmungen ein Wettbewerb zwischen Gemeinwesen geworden.

\subsubsection{Liberalisierung als Antwort}

Von diesem Standortwettbewerb blieben auch der Staat und der Infrastrukturbereich der Schweiz nicht ver- schont. Die öffentliche Verwaltung und die öffentlichen Betriebe wurden zur Leistungsverbesserung und Effizienzsteigerung gezwungen. Als eine - vielleicht die geeignetste - Massnahme zur Verbesserung der Standortattraktivität eines Landes oder einer Region kann die Liberalisierung gelten. Darunter sei hier verstanden, dass der öffentliche Bereich zugunsten des privaten zurückgedrängt, gewissermassen die Trennlinie zwischen Staat und Privat verschoben wird und im öffentlichen Bereich privatwirtschaftliche Elemente eingebaut werden.

Dabei ist es zweckmässig, drei Stossrichtungen der Liberalisierung zu unterscheiden (vgl. Abb. l):

- Die rechtlich-organisatorische Privatisierung besteht in der Überführung einer bis anhin staatlichen Institution in eine private. Anstelle von öffentlichem Recht gilt Privatrecht (OR, ZGB usw.). Denkbar sind auch Zwischenformen (gemischtwirtschaftliche Institutionen). Ausschlaggebend ist letztlich nicht so sehr die Rechtsform als solche, sondern dass die Entscheidungsprozesse unbïrokratisch und flexibel ablaufen und die Organisation entsprechende Entscheidungen erlaubt (Profitcenter und Lean Management).

- Die wirtschaftliche Liberalisierung ist darauf ausgerichtet, dem Wettbewerb als Steuerungs- und Anreizsystem einen grösseren Stellenwert zukommen zu lassen. Wichtig ist der davon ausgehende Effizienzund Innovationsdruck. Das New Public Management (NPM) und das Outsourcing stellen die bekanntesten Formen der wirtschaftlichen Privatisierung dar. Eine Zwischenform zwischen dem staatlichen Monopol und dem vollständig freien Wettbewerb stellt die staatliche Regulierung dar.

- Die finanzielle Privatisierung bringt Änderungen bei der Kostenanlastung. An die Stelle von Steuern treten Preise als Entgelt für die Inanspruchnahme von privaten oder öffentlichen Leistungen. Als Zwischenform können Gebühren und Beiträge betrachtet werden. Derartige Abgaben nach dem Äquivalenzoder dem Verursacherprinzip bewirken ein anderes Verhalten der Nutzer von Leistungen. Waren oder Dienstleistungen werden nicht mehr als gratis empfunden und als Folge davon nicht mehr übernutzt oder verschwendet.

Es ist durchaus möglich, dass diese drei Formen der Liberalisierung unterschiedlich kombiniert werden. Die vollständige Liberalisierung bedeutet somit die Kombination von rechtlich-organisatorischen, wirtschaftlichen und finanziellen Liberalisierungselementen bis zu den jeweiligen Extremen (Teilwürfel links unten in der Abbildung). 


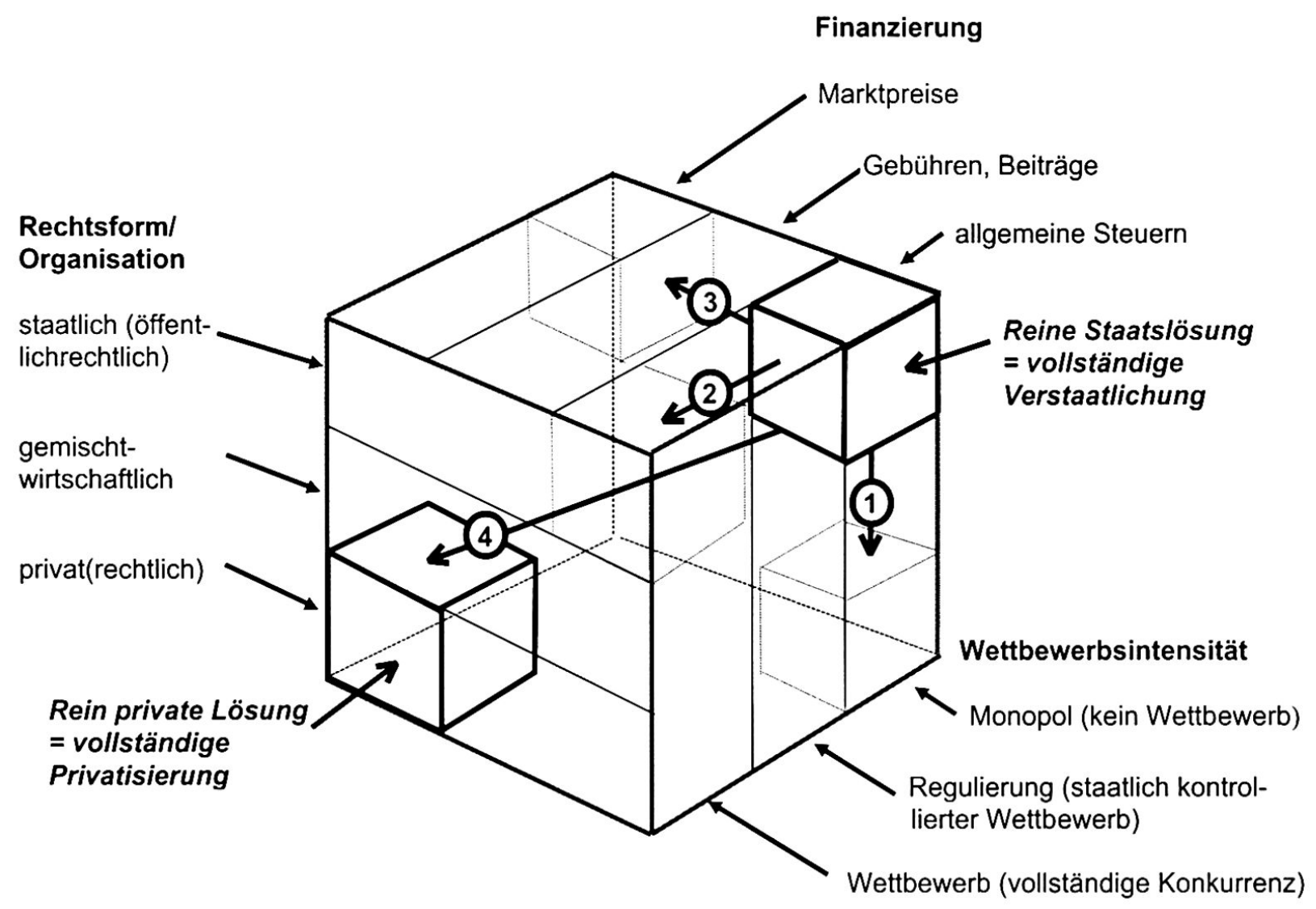

Legende / Key:

(1) Rechtlich-organisatorische Privatisierung / Legal-administrative privatisation

$\Rightarrow$ Flexibilität in Rechtsform, Organisation und Entscheidungsprozessen /

Flexibility of legal status, organisation and decision-making processes

(2) Wirtschaftliche Liberalisierung / Economic liberalisation

$\Rightarrow$ Marktöffnung, Konkurrenz /

free market, competition

(3) Finanzielle Privatisierung / Financial privatisation

$\Rightarrow \quad$ Äquivalenzprinzip, ev. Verursacherprinzip (Kostenwahrheit, Internalisierung von externen

Kosten) /

principle of equivalence, possibly causal principle (cost transparency, internalisation of external costs)

(4) Vollständige Privatisierung / Total privatisation

$=$ rechtlich-organisatorische + wirtschaftliche + finanzielle Privatisierung $/$

legal-administrative + economic + financial privatisation

Abb. 1: Liberalisierungswürfel

Liberalisation cube

Clé de la libéralisation 
Die vollständige Liberalisierung dürfte im Infrastrukturbereich in der Realität eher selten in Frage kommen. In der Regel gibt es nämlich gute Gründe für eine gewisse staatliche Einflussnahme. Auf den ersten Blick paradoxerweise, aber wegen der Gefahr ruinöser Konkurrenz verständlich, können die rechtlich-organisatorische und die finanzielle Privatisierung sogar mit einer Verstärkung der Regulierung verbunden sein, wenn es darum geht, bestimmte übergeordnete Anliegen zu verwirklichen. Solche Anliegen können sein:

- die Verhinderung von privaten Monopolen (Sicherstellung eines wirksamen, effizienten und fairen Wettbewerbs).

- die Sicherstellung einer bestimmten Grundversorgung für alle Bevölkerungsgruppen und Landesteile (sog. service public).

- Gerechtigkeitsziele (Abbau von Einkommensunterschieden zwischen Personengruppen, Sektoren, Regionen usw.).

- ökologische Ziele (Umwelt- und Landschaftsschutz, Erhaltung der Biodiversität, Sicherstellung einer nachhaltigen Entwicklung usw.).

\section{Privatisierung von vier Infrastrukturbereichen}

\subsection{Schienenverkehr}

Bis Ende 1998 waren die SBB ein Bundesbetrieb. Dieser wurde in den letzten rund zehn Jahren nur in kleinen Schritten mit privatwirtschaftlichen Elementen angereichert. Zwei Vorstellungen hemmten die weitergehende Privatisierung: erstens, dass der service public einen staatlichen Monopolbetrieb bedinge, zweitens, dass Infrastruktur und Betrieb in einer Hand sein müssten. Ähnliches gilt für die sogenannten Privatbahnen (konzessionierte Transportunternehmungen KTU). In finanzieller Hinsicht sind alle Bahnen (mit Ausnahme gewisser touristischer Bahnen) in starkem Ausmass auf Steuermittel angewiesen (Übernahme von Infrastrukturlasten und Abgeltung gemeinwirtschaftlicher Leistungen durch den Staat, vor allem den Bund).

Unter dem Druck der Liberalisierung in der EU und den Nachbarländern, der Konkurrenz durch den Strassenverkehr sowie der defizitären Bundesfinanzen erfolgte auf Anfang 1999 eine grundlegende Reform: neues Eisenbahngesetz, neues SBB-Gesetz, Reorganisation der SBB (SBB AG mit dem Bund als alleinigem Aktionär), Netzzugang für Dritte (free access), Wettbewerb im Regionalverkehr (Ausschreibung von Bahnlinien), Trennung von Infrastruktur und Betrieb, Entschuldung der SBB (Beseitigung von Altlasten), neue
Investitionsfinanzierung, Bestellerprinzip bei nicht rentablen Linien, Leistungsvereinbarung, Abschaffung des Beamtenstatus usw. Die Konzessionserteilung und Überwachung der neuen «Spielregeln» erfolgt durch das Bundesamt für Verkehr als von den Bahngesellschaften unabhängige Instanz.

\subsection{Elektrizität}

Von den vier hier untersuchten Infrastrukturbereichen ist die Elektrizitätswirtschaft in der Vergangenheit am wenigsten verstaatlicht gewesen. Sie ist dem gemischtwirtschaftlichen Bereich zuzurechnen. Die auch diesen Sektor ergreifende Liberalisierung nimmt daher weniger die Form der Privatisierung als der Deregulierung an. Dabei ist zu unterscheiden zwischen dem Hochspannungsnetz (heute sieben Verbundgesellschaften als «Strom-Grossisten») und dem Verteilungsnetz (rund 1000, z.T. sehr kleine, meist kommunale Gesellschaften als «Strom-Detaillisten»). Zur Diskussion stehen auf der einen Seite die Schaffung von ein bis zwei schweizerischen Netzgesellschaften, auf der anderen Seite der Zusammenschluss der vielen Verteilgesellschaften zu grösseren Unternehmungen.

Auslösender Faktor für die Liberalisierung war der Entscheid der EU, den Strommarkt ab dem 19. Februar 1999 schrittweise zu öffnen, das heisst, den diskriminierungsfreien Netzzugang - zunächst für Grossverteiler und Grossverbraucher - zu gewährleisten. Grundsätzlich will und wird die Schweiz mitmachen, allein schon um die Reziprozität, das heisst, den Zugang schweizerischer Elektrizitätsgesellschaften zu ausländischen Märkten, zu gewährleisten.

Im Vergleich namentlich zu Grossbritannien und den nordischen Ländern ist die Schweiz insbesondere bezüglich Marktöffnung im Hintertreffen. Die Elektrizitätswirtschaft verweist auf das ungelöste Problem der nicht-amortisierten Investitionen (NAI) und fordert für den Fall einer raschen Marktöffnung vom Bund Abgeltungen in Milliardenhöhe.

\subsection{Telekommunikation}

Jahrzehntelang waren in Europa die Post und die Telekommunikation in staatlichen Unternehmungen vereinigt. Die Aufspaltung der PTT-Betriebe erfolgte in der Schweiz schrittweise, zunächst durch Verselbstständigung des Telekommunikationsbereichs. Anstoss gaben entsprechende Liberalisierungsschritte in den USA (Aufbrechung des privaten Monopols AT\&T) und in England (Privatisierung der British Telecom), Marktöffnungsbeschlüsse der EU sowie geradezu sprunghafte Innovationen im technologischen $\mathrm{Be}$ reich durch die Verschmelzung von Telekommunikation und Informatik. Die Liberalisierung setzte bei den Endgeräten an. Es folgten die Mehrwertdienste. 
Ein eigentlicher «big bang» erfolgte mit der «mutigsten» Privatisierung der Schweiz: der Gründung von Swisscom Anfang 1998 und dem Börsengang im Herbst 1998, der Schaffung von Wettbewerb durch Zulassung von Konkurrenten sowie der Ermöglichung des Netzzugangs (Interkonnektion). Während einer Übergangszeit von ein paar Jahren hat Swisscom noch eine Versorgungspflicht. Sie erhält hierfür vom Staat keine Abgeltung. Die Begründung lautet: Swisscom verfüge als Nachfolgeunternehmung der PTT über gewisse Privilegien. Weitere Regulierungen betreffen die Preisobergrenze, die freie Netzwahl der Kunden und die Nummernportabilität. Als Regulierungsbehörden fungieren die Wettbewerbskommission, der Preisüberwacher, das Bundesamt für Kommunikation (Bakom) und die Kommunikationskommission (ComCom).

Es ist verfrüht, die Liberalisierung im Telekommunikationsbereich definitiv zu beurteilen. Immerhin hat die Intensivierung des Wettbewerbs bereits eine deutliche Verbesserung der Angebotspalette und eine Senkung der Preise zur Folge gehabt. Aus «Untertanen» der ehemaligen PTT, die lediglich zwischen schwarzen und grauen Telefonapparaten wählen konnten und erst noch zur Miete verpflichtet waren, sind Kundinnen und Kunden geworden, die von heftig konkurrierenden Anbietern umworben werden.

\subsection{Post}

Die Aufspaltung der früheren PTT in einen Post- und einen Telekommunikationsbereich hat - auch unter dem Druck ausländischer Konkurrenz (vor allem bei der Paketpost und den Kurierdiensten) - für die Post einige Veränderungen ergeben. Unter anderem ist die Quersubventionierung der Post aus Überschüssen der Telekommunikationsdienste hinfällig geworden. Eine Quersubventionierung besteht allenfalls noch zwischen Postfinance und der eigentlichen Post.

Bei der Aufspaltung der PTT sind für den Postbereich im Vergleich zu den anderen Infrastrukturbereichen die kleinsten Privatisierungsschritte gemacht worden. Die Post erhielt Anfang 1998 einen deutlich grösseren unternehmerischen Spielraum. Sie ist eine öffentlichrechtliche Anstalt, die sich wie eine private Unternehmung organisieren kann und die Möglichkeit hat, ihr Sortiment weitgehend selbst zu bestimmen.

Die Post geniesst im Briefbereich nach wie vor einen Monopolschutz. Als Gegenleistung hat die Post Auflagen bei der Versorgung (flächendeckende Poststellen und Briefzustellung) zu beachten. Sie ist zur Eigenwirtschaftlichkeit verpflichtet und soll auch einen Teil ihrer Investitionen selbst finanzieren.

Der Handlungsspielraum der Post wird (im Unterschied zur SBB AG) durch Altlasten behindert. Zudem hat sie es (im Unterschied zu Swisscom) in ihrem Kernbereich mit einem tendenziell schrumpfenden Markt zu tun. Die klassische Briefpost wird zunehmend durch die elektronische Post (Fax und E-Mail) ersetzt. Die Post ist daher auf der Suche nach neuen Produkten und neuen Märkten. Solche gibt es, beispielsweise in Form integrierter Dienste und des E-Commerce.

Während ausländische Konkurrenten über die erforderlichen Mittel verfügen, um in grossem Stile Firmen aufzukaufen - zum Beispiel hat die Deutsche Post das weltweit tätige Schweizer Speditionsunternehmen Danzas erworben -,ist die Schweizer Post diesbezüglich stark eingeschränkt und auf Allianzen angewiesen. Es würde nicht überraschen, wenn es auch hier zu Fusionen und Übernahmen kommt und die Schweizer Post in ihrem angestammten Bereich langfristig vom Markt verdrängt wird oder ihre Selbstständigkeit verliert.

\subsection{Beurteilung}

Die Analyse des Stands der Liberalisierung in den Infrastrukturbereichen Verkehr, Energie, Telekommunikation und Post hat deutliche Unterschiede gezeigt. Diese sind in in der folgenden Übersicht (Abb. 2) schematisch anhand der drei Liberalisierungsdimensionen dargestellt.

Vereinfachend lässt sich für die jüngere Vergangenheit feststellen $(\mathrm{A} \rightarrow \mathrm{B})$, dass

- die kleinsten Veränderungen bei der Finanzierung gemacht worden sind. Dies erklärt sich damit, dass namentlich bei der Elektrizität und bei der Telekommunikation schon seit langem Eigenwirtschaftlichkeit angestrebt und erreicht wird. Nach erfolgter Liberalisierung wird jedoch das Gewinnmotiv an Bedeutung gewinnen, weil neu die Aktionäre hinzukommen, deren Ansprüche zu befriedigen sind.

- die grössten Liberalisierungsschritte bei der Intensivierung des Wettbewerbs getan worden sind. Die wirtschaftliche Liberalisierung äussert sich vor allem in der Marktöffnung für Konkurrenten und in der erhöhten Wahlfreiheit für die Konsumentinnen und Konsumenten.

- deutliche Unterschiede bei der rechtlich-organisatorischen Privatisierung bestehen, das heisst beim Übergang von der öffentlich-rechtlichen zur privatrechtlichen Normierung.

- klar zwischen Infrastrukturnetz und Infrastrukturbetrieb unterschieden werden muss. Je weiter die Privatisierung geht, desto wichtiger wird im Netzbereich die Regulierung, dies angesichts der Gefahr, dass bei natürlichen Monopolen sonst der Wettbewerb zum Erliegen kommt. 


\begin{tabular}{|c|c|c|c|}
\hline \multirow{3}{*}{ Bereich } & \multicolumn{3}{|c|}{ Liberalisierung } \\
\hline & Rechtlich-organisatorisch & Wirtschaftlich & Finanziell \\
\hline & staatlich privat & staatlich & staatlich \\
\hline $\begin{array}{l}\text { Schienen- } \\
\text { verkehr }\end{array}$ & $A \rightarrow B \rightarrow C$ & $A \longrightarrow B \rightarrow C$ & $A \rightarrow B \rightarrow C$ \\
\hline Elektrizität & $A B \rightarrow C$ & $A \rightarrow B \rightarrow C$ & $A B C$ \\
\hline $\begin{array}{l}\text { Telekommu- } \\
\text { nikation }\end{array}$ & $\mathrm{A} \longrightarrow \mathrm{BC}$ & $A \longrightarrow B C$ & $A B C$ \\
\hline Post & $A \rightarrow B \rightarrow C$ & $\mathrm{~A} \rightarrow \mathrm{B} \rightarrow \mathrm{C}$ & $A \rightarrow B C$ \\
\hline
\end{tabular}

\section{A Ausgangssituation (vor etwa 10 Jahren) \\ B Gegenwärtige Situation \\ C Längerfristig anzustrebende Situation}

Abb. 2: Entwicklung der Liberalisierung Liberalisation as a process of development Développement de la libéralisation

Die eingeleiteten Privatisierungsmassnahmen gehen unter dem Gesichtspunkt der Verbesserung der internationalen Wettbewerbsfähigkeit der schweizerischen Volkswirtschaft zweifellos in die richtige Richtung, jedoch teilweise noch zu wenig weit. Vermutlich werden in den nächsten Jahren der Marktdruck wie auch der äussere politische Druck weitere Massnahmen zur Effizienz- und Innovationssteigerung erzwingen $(B \rightarrow C)$. Der grösste verbleibende Handlungsbedarf besteht bei den SBB im finanziellen Bereich und bei der Post bei der rechtlichen Verselbstständigung.

\section{Konsequenzen für die Raumordnungspolitik}

Die Privatisierung und Deregulierung in den vier untersuchten Infrastrukturbereichen bedeutet Folgendes:

- Der Staat (Bund) wird vom Macher zum Überwacher.

- Er hat für den Infrastrukturbereich die Wettbewerbsregeln aufzustellen und deren Einhaltung zu kontrollieren.

- Er hat die Internalisierung der externen Kosten anzustreben, um sicherzustellen, dass die markt- wirtschaftliche Steuerung zu optimalen Ergebnissen führt und Nachhaltigkeit erreicht wird.

Beides führt zu einer gewissen Re-Regulierung.

- Wettbewerbsfähigkeit wird zur dominanten Handlungsmaxime. Die Infrastrukturunternehmungen SBB AG, Elektrizitätsgesellschaften, Swisscom (und ihre Konkurrenten) sowie Post haben - nach einer Übergangszeit - nur noch die Aufgabe der bedïrfnisgerechten und kostengïnstigen Versorgung der Bevölkerung mit ihren Produkten.

- Effizienzanliegen auf der einen Seite sowie Gerechtigkeits- und Ausgleichsanliegen auf der anderen Seite werden getrennt.

- Wenn von den Infrastrukturunternehmungen für sie nicht rentable Leistungen im Bereich der Grundversorgung (Service public) verlangt werden, so müssen diese auf vertraglicher Basis sichergestellt und die entsprechenden Mehrkosten durch den Staat abgegolten werden. Erst recht gilt dies für ausländisch kontrollierte Unternehmungen.

- Bei der grossräumigen Netzinfrastruktur sind rein nationale Lösungen überholt. 
Für die Raumordnungspolitik bedeuten diese Veränderungen:

- Die Infrastruktur steht ihr langfristig nicht mehr als Instrument zur Verfügung. Zumindest gilt dies für die vier untersuchten Bereiche und auf mittlere Frist. Während einer Übergangszeit können im Sinne des Service public den Infrastrukturunternehmungen noch gewisse Auflagen gemacht werden.

- Die Sicherstellung der Grundversorgung, das heisst «die Bereitstellung von Waren und Dienstleistungen von einem speziellen öffentlichen Interesse für alle Bevölkerungsschichten und Wirtschaftsunternehmungen in allen Landesteilen zu einem günstigen (erschwinglichen) Preis», muss durch die dafür zuständigen Gemeinwesen bestellt, und die Mehrkosten müssen aus Steuermitteln abgegolten werden.

- Wenn über den Bau und Betrieb von Infrastruktureinrichtungen Standortpolitik (Implantation von standortgebundenen Einrichtungen als «Magneten» für die wirtschaftliche Entwicklung einer Region) oder Regionalpolitik (im Sinne des Ausgleichs zwischen wirtschaftlich starken und wirtschaftlich schwachen Regionen) betrieben werden soll, so bedingt dies entsprechende Verträge mit den Infrastrukturunternehmungen und Vergütung der Mehrkosten (Besteller-Bezahler-Prinzip).

- Dies hat den Vorteil, dass die tatsächlichen Kosten der Raumordnungspolitik offen ausgewiesen werden. Durch die Kostenwahrheit wird der Druck vergrössert, die jeweils zweckmässigsten und kostengünstigsten Massnahmen zu ergreifen.

- Regionaler Ausgleich muss in Zukunft vor allem über den freien Finanzausgleich erfolgen. Es ist daher wichtig, dass der Finanzausgleich auf eine neue Grundlage gestellt wird und dabei ebenfalls klar zwischen Umverteilungs- und Effizienzanliegen unterschieden wird. Dies ist im Übrigen die Grundidee des Reformprojekts «Neuer Finanzausgleich». Man kann den Finanzausgleich auch als nachträgliche Umverteilung von Effizienzgewinnen von wirtschaftsstarken Unternehmungen und Personen, die von der Globalisierung und Liberalisierung profitieren, zu wirtschaftsschwachen Regionen und Kantonen interpretieren. In welchem Umfang dies geschehen soll, ist eine politische Frage.

Der Privatisierungsprozess wird sich vermutlich noch über viele Jahre erstrecken. Dies erlaubt die durchdachte Neuorientierung der Raumordnungspolitik und deren schrittweise Umsetzung.

\section{Literatur}

FrEY, R.L. (1979): Die Infrastruktur als Mittel der Regionalpolitik. Eine wirtschaftstheoretische Untersuchung zur Bedeutung der Infrastrukturförderung von entwicklungsschwachen Regionen in der Schweiz. - Bern/Stuttgart: Haupt.

FREY, R.L. (1999): Liberalisierung und Privatisierung in den Infrastrukturbereichen Verkehr, Energie, Telekommunikation. Bericht zuhanden des Bundesamtes für Wirtschaft und Arbeit vom Januar 1999. - Abgedruckt in Kommission für Konjunkturfragen: Liberales wirtschaftspolitisches Konzept, Materialband, Bern: KfK.

INFRAS in Zusammenarbeit mit OetTERLI, J. (1998): Liberalisierung und Grundversorgung in den Sektoren Telekommunikation, Post, Elektrizität und öffentlicher Verkehr. State-of-the-Art Bericht und sektorübergreifende Übersicht. - Bericht zuhanden des Bundesamtes für Wirtschaft und Arbeit und des Bundesamtes für Raumplanung vom 14.12.98.

KAHN,A.E. (1988):The Economics of Regulation. Principles and Institutions. - Cambridge/Mass./London: MIT Press.

Kaufer, E. (1981): Theorie der Öffentlichen Regulierung. - München: Vahlen.

OECD (1995): Telecommunication Infrastructure: The Benefits of Competition. - Paris: OECD.

SHLEIfER, A. (1998): «State versus Private Ownership». - In: Journal of Economic Perspectives 12: 133-150.

von Weizsäcker, C.C. (1982): «Staatliche Regulierung - positive und normative Theorie». - In: Schweizerische Zeitschrift für Volkswirtschaft und Statistik 118. Jg.: 325-341.

\section{Zusammenfassung: Privatisierung der Infrastruktur und Raumordnungspolitik}

Die Globalisierung und der internationale Standortwettbewerb haben in den letzten Jahren auch in der Schweiz eine eigentliche Liberalisierungs-, Privatisierungs- und Deregulierungswelle ausgelöst. Dadurch wurden in den davon betroffenen Infrastrukturbereichen (Eisenbahn, Post, Telekommunikation, Elektrizitätswirtschaft) beachtliche Innovationsschübe ausgelöst. Eine zunächst kaum beachtete Nebenwirkung besteht darin, dass der Staat Instrumente verloren hat, um raumordnungspolitische Ziele zu verwirklichen. Wenn über den Bau und Betrieb von Infrastruktureinrichtungen Standortpolitik oder Regionalpolitik betrieben werden soll, so bedingt dies in Zukunft den Abschluss entsprechender Verträge mit den Infrastrukturunternehmungen und die Vergütung der Mehrkosten durch den Staat. Der regionale Ausgleich wird vor allem über den freien Finanzausgleich erfolgen müssen. 
Résumé: Privatisation de l'infrastructure et politique d'aménagement du territoire

En Suisse, la globalisation et la compétition entre les villes, régions et nations ont déclenché une véritable vague de libéralisations, de privatisations et de dérégulations. A sa suite, des innovations considérables ont été faites dans les domaines de l'infrastructure concernée (chemin de fer, poste, télécommunication, électricité). L'état a perdu quelques-uns de ses instruments de politique d'aménagement du territoire, effet secondaire négligé dans les premiers temps. Si la construction et la gestion des infrastructures doivent encore servir aux fins de la politique de localisation ou de la politique régionale, l'État devra à l'avenir conclure des contrats avec les entreprises d'infrastructure et leur rembourser les coûts supplémentaires. La réduction des disparités régionales sera à réaliser surtout à travers une péréquation financière non affectée.

\section{Summary: Privatisation of Infrastrucure and Regional Policies}

Globalisation and international locational competition have set off a wave of liberalisation, privatisation and deregulation in Switzerland. This resulted in important innovations in the fields concerned (railways, postal services, telecommunication, electricity). As a secondary effect, often neglected at first, the government lost instruments for pursuing regional policy goals. If constructing and operating the infrastructure is still be used to facilitate locational or regional policy, contracts must be negociated with the infrastructure enterprises. The government will have to refund the additional costs and regional redistribution will have to be achieved mainly by federal revenue sharing.

Prof. Dr. René L. Frey, Wirtschaftswissenschaftliches Zentrum WWZ der Universität Basel, Petersgraben 51, CH-4003 Basel. e-mail: Rene.Frey@unibas.ch 